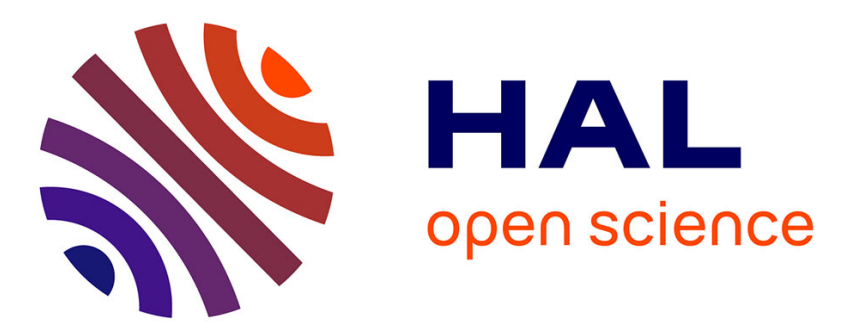

\title{
Modélisation du courant de seuil de diodes laser GaAlAs à guidage par l'indice
}

\author{
Françoise Lozes-Dupuy, B. Saint-Cricq, G. Vassilieff, L. Vassilieff, A.
}

Bensoussan

\section{- To cite this version:}

Françoise Lozes-Dupuy, B. Saint-Cricq, G. Vassilieff, L. Vassilieff, A. Bensoussan. Modélisation du courant de seuil de diodes laser GaAlAs à guidage par l'indice. Revue de Physique Appliquée, 1987, 22 (11), pp.1561-1569. 10.1051/rphysap:0198700220110156100 . jpa-00245708

\section{HAL Id: jpa-00245708 https://hal.science/jpa-00245708}

Submitted on 1 Jan 1987

HAL is a multi-disciplinary open access archive for the deposit and dissemination of scientific research documents, whether they are published or not. The documents may come from teaching and research institutions in France or abroad, or from public or private research centers.
L'archive ouverte pluridisciplinaire HAL, est destinée au dépôt et à la diffusion de documents scientifiques de niveau recherche, publiés ou non, émanant des établissements d'enseignement et de recherche français ou étrangers, des laboratoires publics ou privés. 


\title{
Modélisation du courant de seuil de diodes laser GaAlAs à guidage par l'indice
}

\author{
F. Lozes-Dupuy, B. Saint-Cricq, G. Vassilieff, L. Vassilieff et A. Bensoussan \\ Laboratoire d'Automatique et d'Analyse des Systèmes, 7 avenue du Colonel Roche, 31077 Toulouse Cedex, \\ France
}

(Reçu le 2 février 1987, révisé le 25 mai 1987, accepté le 18 juin 1987)

\begin{abstract}
Résumé. - Le comportement d'une diode laser à ruban VSIS au voisinage du seuil est décrit à partir des modèles généraux du guide optique bidimensionnel, du gain optique du matériau, du comportement électrique de la structure. Les propriétés du guidage sont analysées à partir des variations latérales de l'indice effectif complexe. Les relations gain-niveau de courant sont évaluées pour une même famille d'alliages en se basant sur les propriétés du coefficient de création de paires électron-trou. Le courant total qui traverse la structure est calculé à partir des recombinaisons dans la couche active, du courant de conduction et de diffusion dans les couches de confinement, de la répartition latérale des lignes de courant hors du ruban. L'ensemble de ces modèles s'articule pour proposer une méthodologie générale de détermination du courant de seuil, valable pour la plupart des diodes laser à guidage par l'indice.
\end{abstract}

\begin{abstract}
The threshold of a VSIS laser diode is described using general models for the bidimensional optical guide, the optical gain of the material, the electrical behaviour of the structure. Guiding mechanisms are analysed in relation to the lateral variations of the complex effective index. Gain-injection level relations are evaluated for a whole alloy family, using the electron-hole pair generation coefficient. The total current through the structure is given by the recombinations in the active layer, the drift and diffusion current in the confining layers, the spreading current on either side of the stripe. The different models are well linked to establish a general methodology leading to the threshold current determination of most index guided laser diodes.
\end{abstract}

\section{Introduction.}

Les diodes laser, composants clef des systèmes optoélectroniques, sont l'objet d'un important effort de recherches qui porte notamment sur :

- l'élargissement du domaine d'émission, par la mise au point de nouvelles filières de matériaux,

- l'évaluation des limites de fonctionnement des dispositifs actuels,

- l'optimisation des performances par une meilleure conception de la structure ou grâce à une maîtrise croissante des processus technologiques.

Dans tous les cas, le courant de seuil de la diode est un paramètre dont la valeur est critique pour l'obtention de l'émission laser, le fonctionnement continu ou pulsé, l'intégrabilité, la fiabilité, etc...

Il apparaît nécessaire de disposer d'une méthodologie générale de détermination du courant de seuil qui lie les différents paramètres technologiques aux caractéristiques de la cavité oscillante, au confinement électrique et optique, aux propriétés optiques du matériau de la couche active.

L'objectif de cet article est de présenter, sur un exemple, le principe des différentes étapes de calcul du courant de seuil d'une diode laser. La diode choisie est représentative des diodes laser à guidage par l'indice, connues comme les plus performantes parmi les diodes laser à ruban. Il s'agit de la diode à ruban VSIS (V Channelled Substrate Inner Stripe) [1], dont la double hétérojonction, qui réalise le confinement transverse, est épitaxiée sur une couche bloquante gravée assurant le confinement latéral simultané de la lumière et des lignes de courant (voir Fig. 1).

La modélisation de ce type de structure est établie en prenant en compte les dimensions, compositions et dopages des couches principales et en les reliant aux mécanismes physiques qui régissent le seuil d'émission laser. Elle décrit : 


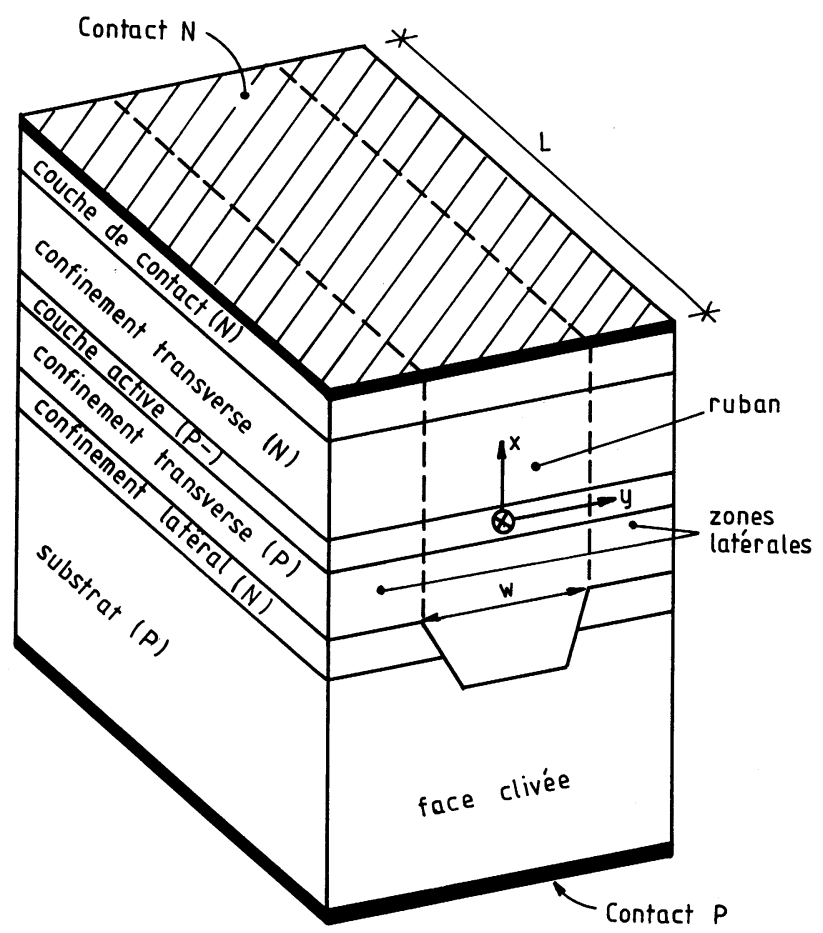

Fig. 1. - Représentation schématique d'une diode laser à ruban VSIS.

[Schematic VSIS laser structure.]

- le guidage bidimensionnel de la lumière,

- les propriétés de gain optique de la couche active,

- le comportement électrique dans le ruban et dans les zones latérales.

Il est montré ensuite comment s'articulent ces trois modèles pour constituer une méthodologie générale de détermination du courant de seuil de la diode.

\section{Guide optique bidimensionnel.}

La fraction de lumière confinée dans la couche active du ruban résulte d'un guidage bidimensionnel :

- le confinement optique transverse est réalisé par l'empilement des couches, qui constitue un guide multicouches, généralement monomode comptetenu de l'épaisseur de la couche active, dont les propriétés peuvent être décrites par la méthode classique de l'indice effectif [2].

- le confinement optique latéral est obtenu par variation latérale de cet indice effectif.

Il faut noter que la prise en compte des mécanismes de gain et d'absorption impose la description de chaque couche par son indice de réfraction complexe défini par :

$$
\bar{n}=n-j \frac{\alpha}{2 k_{0}}
$$

$n$ étant l'indice de réfraction de l'alliage, $\alpha$ l'absorption (le gain $g$ étant son opposé), $k_{0}$ la constante de propagation dans le vide. La propagation dans le guide est alors obtenue par la résolution des équations de Maxwell sous la forme la plus générale en utilisant le formalisme de l'indice effectif complexe.

Les diodes laser à ruban VSIS présentent un guidage par l'indice basé sur la variation d'épaisseur de la couche de confinement entre le substrat et la couche active. Dans les zones latérales, le substrat est suffisamment proche de la couche active pour absorber la lumière émise et conduire à un indice effectif latéral inférieur à celui du ruban.

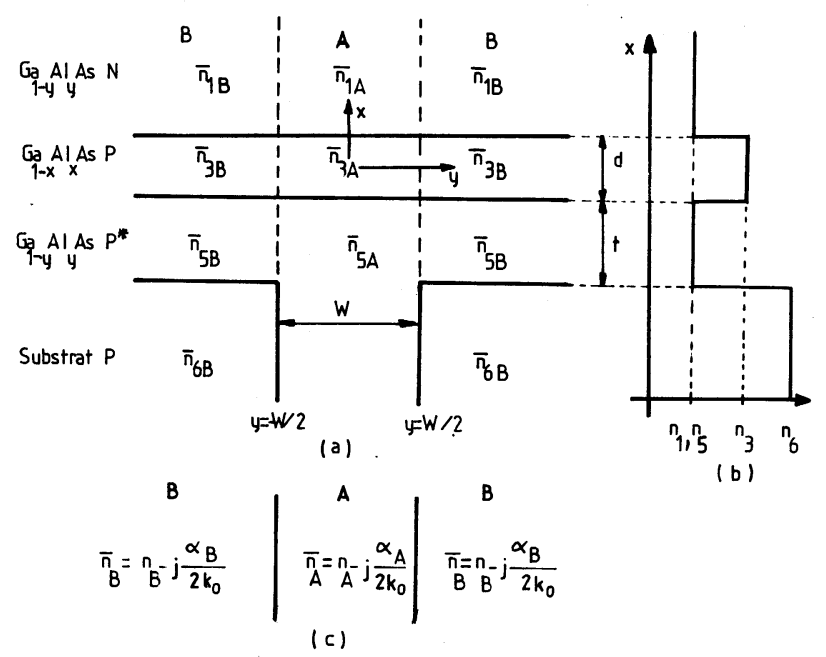

Fig. 2. - a) Représentation du guide VSIS. b) Profil d'indice suivant la direction $x$. c) Guide unidimensionnel à partir des confinements équivalents.

(a) Configuration of the VSIS waveguide. b) Refractive index profile along the $x$ direction. c) 1-D guide with equivalent confinement.]

L'ensemble du guide VSIS se décompose en trois régions comme indiqué sur la figure 2 et s'analyse selon les étapes suivantes :

- la région de ruban $\mathrm{A}$, de largeur égale à celle du sillon, est considérée comme un guide de lumière à trois couches dans la direction $x$, dont on détermine l'indice effectif complexe $\overline{n_{\mathrm{A}}}$ du mode TE,

- les régions latérales $B$ sont considérées comme deux guides de lumière à quatre couches dans la direction $x$, dont on détermine l'indice effectif complexe $\overline{n_{\mathrm{B}}}$ du mode TE,

- la propagation dans le guide VSIS est étudiée à partir des modes TM du guide à trois couches dans la direction $y$, l'indice de la zone centrale étant égal à $\overline{n_{\mathrm{A}}}$ et celui des couches latérales à $\overline{n_{\mathrm{B}}}$.

La résolution des équations de Maxwell [3] dans chaque guide (A, B, VSIS) permet, à partir des dimensions et des indices complexes des différentes couches, de déterminer en particulier, pour chaque 
mode du guide VSIS, la constante de propagation complexe, l'indice effectif $n_{\mathrm{m}}$ et le gain modal $g_{\mathrm{m}}$, la fraction de lumière $\Gamma$ confinée dans la couche active du ruban.

La détermination du seuil laser impose de connaître le gain $g_{3 \mathrm{~A}}$ qui doit être atteint par le matériau de la couche active pour que le gain modal $g_{\mathrm{m}}$ du guide VSIS égale le gain $\boldsymbol{g}_{\text {cav }}$ fixé par la condition d'oscillation de la cavité. La valeur du gain $g_{3 \mathrm{~A}}$ s'obtient par un calcul itératif en résolvant successivement les équations de Maxwell dans les différents guides, selon l'organigramme de la figure 3.

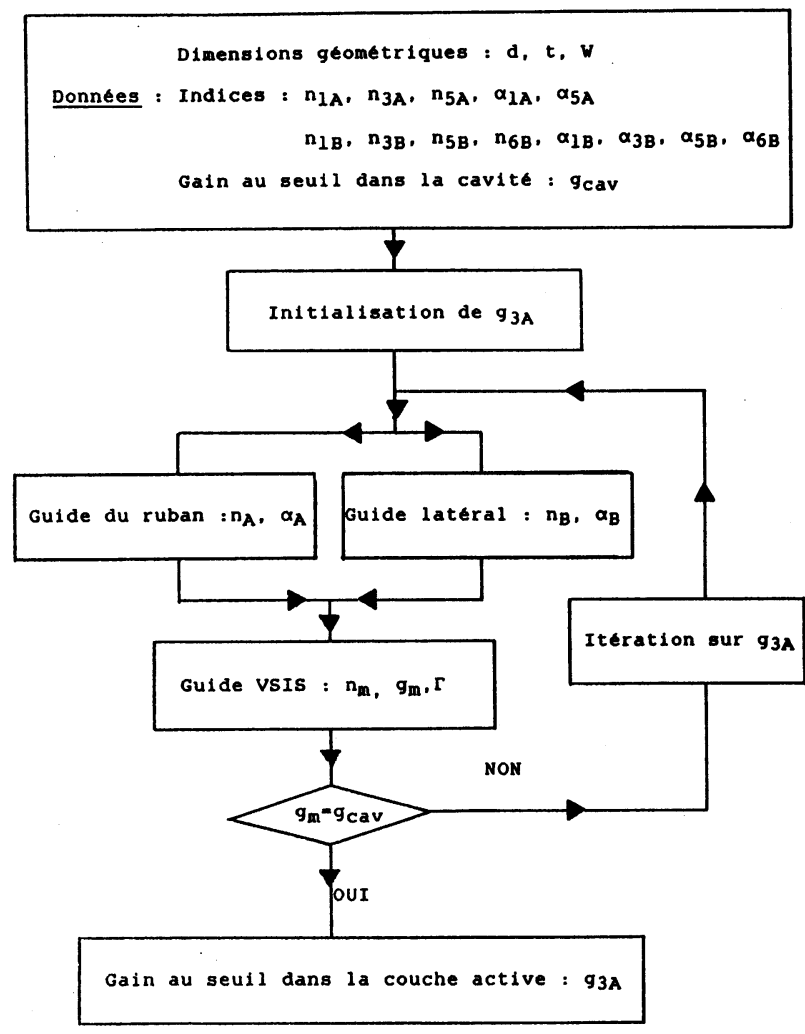

Fig. 3. - Détermination du gain de la couche active.

[Flow chart for gain determination in the active region.]

\section{Evaluation du gain optique dans une famille d'alliages.}

Les propriétés optiques des alliages à structure de bande directe, susceptibles de constituer la couche active d'une diode laser, restent dans l'ensemble encore mal connues et n'ont été l'objet que de peu d'études exhaustives [4,5].

Le but de ce paragraphe est de présenter une méthode d'évaluation du gain optique dans une famille d'alliages, à partir de résultats expérimentaux obtenus sur un élément de cette famille : l'exemple proposé concerne les alliages GaAlAs mais son principe pourrait être mis à profit pour une autre famille d'alliages.
Les variations du coefficient de gain avec l'énergie du photon et le niveau de polarisation ont été déterminées théoriquement dans le cas du GaAs, à partir du calcul des densités d'états et de la matrice optique $[6,7]$. Cette procédure exige une connaissance approfondie de la structure de bande du matériau et sa complexité la rend difficilement généralisable à l'étude de l'ensemble des alliages GaAlAs.

En l'absence de données expérimentales, les spectres de gain des alliages GaAlAs peuvent être évalués par extrapolation des mesures obtenues pour le GaAs [8], en se basant sur les propriétés du coefficient $\alpha^{\prime}$ de création de paires électron-trou.

Ce coefficient est défini par :

$$
\begin{aligned}
\alpha^{\prime}(E)= & \frac{q^{2} \hbar \pi}{m_{\mathrm{n}}^{2} \varepsilon_{0} c n E} \int_{-\infty}^{+\infty}\left|M\left(E^{\prime}, E^{\prime \prime}\right)\right|^{2} \rho_{\mathrm{C}}\left(E^{\prime}\right) \times \\
& \times \rho_{\mathrm{V}}\left(E^{\prime \prime}\right) f_{\mathrm{V}}\left(E^{\prime \prime}\right)\left(1-f_{\mathrm{C}}\left(E^{\prime}\right)\right) \mathrm{d} E^{\prime}
\end{aligned}
$$

où $M$ est la matrice optique associée à la transition $\left(E^{\prime}-E^{\prime \prime}\right)$ d'énergie $E, \rho_{\mathrm{C}}, f_{\mathrm{C}}, \rho_{\mathrm{V}}, f_{\mathrm{V}}$ représentent respectivement la densité d'états et la probabilité d'occupation dans la bande de conduction et de valence, $n$ est l'indice de réfraction, $\varepsilon_{0}$ la permittivité du vide, $m_{\mathrm{n}}$ la masse effective de l'électron.

Les coefficients de gain $g(E)$ et d'absorption $\alpha(E)$, qui rendent compte du bilan entre les mécanismes contraires de création de paires électron-trou et d'émission stimulée, se déduisent, pour un écart de pseudo-niveaux de Fermi $\left(E_{\mathrm{FN}}-E_{\mathrm{FP}}\right)$ par les relations :

$$
\begin{aligned}
g(E) & =-\alpha(E) \\
& =\alpha^{\prime}(E)\left(\exp \frac{\left(E_{\mathrm{FN}}-E_{\mathrm{FP}}\right)-E}{k T}-1\right) .
\end{aligned}
$$

De même, on montre que le taux total d'émission spontanée s'exprime à partir du coefficient de création de paires électron-trou :

$$
\begin{aligned}
R_{\text {spon }}=\frac{8 \pi}{h^{3}} \frac{n^{2}}{c^{2}} \exp & \frac{E_{\mathrm{FN}}-E_{\mathrm{FP}}}{k T} \times \\
& \times \int_{0}^{\infty} \alpha^{\prime}(E) E^{2} \exp \frac{-E}{k T} \mathrm{~d} E .
\end{aligned}
$$

Ce dernier se met sous la forme :

$$
R_{\mathrm{spon}}=\beta \exp \frac{\left(E_{\mathrm{FN}}-E_{\mathrm{FP}}\right)}{k T}
$$

en posant

$$
\beta=\frac{8 \pi}{h^{3}} \frac{n^{2}}{c^{2}} \int_{0}^{\infty} \alpha^{\prime}(E) E^{2} \exp \frac{-E}{k T} \mathrm{~d} E .
$$

Les variations expérimentales du coefficient $\alpha^{\prime}(E)$, relevées par Henry et al. [9] sur du matériau GaAs ont été choisies comme base d'extrapolation 
pour les alliages $\mathrm{Ga}_{1-x} \mathrm{Al}_{x} \mathrm{As}(x<0,4)$, en suivant un principe analogue à celui utilisé pour le coefficient d'absorption [10]. Ces mesures, effectuées in situ sur des diodes laser, sont représentatives pour les densités de porteurs caractéristiques du seuil laser $\left(n_{\Gamma} \# 10^{18} \mathrm{~cm}^{-3}\right.$ dans la vallée directe $\Gamma$ ) et rendent compte par suite des effets de réduction de bande interdite et d'interactions entre porteurs sur les mécanismes d'absorption. La courbe de référence $(x=0)$ et les courbes extrapolées sont portées sur la figure 4. Le figure 5 donne un exemple de spectres de gain déduits de ces variations et de la relation (3).

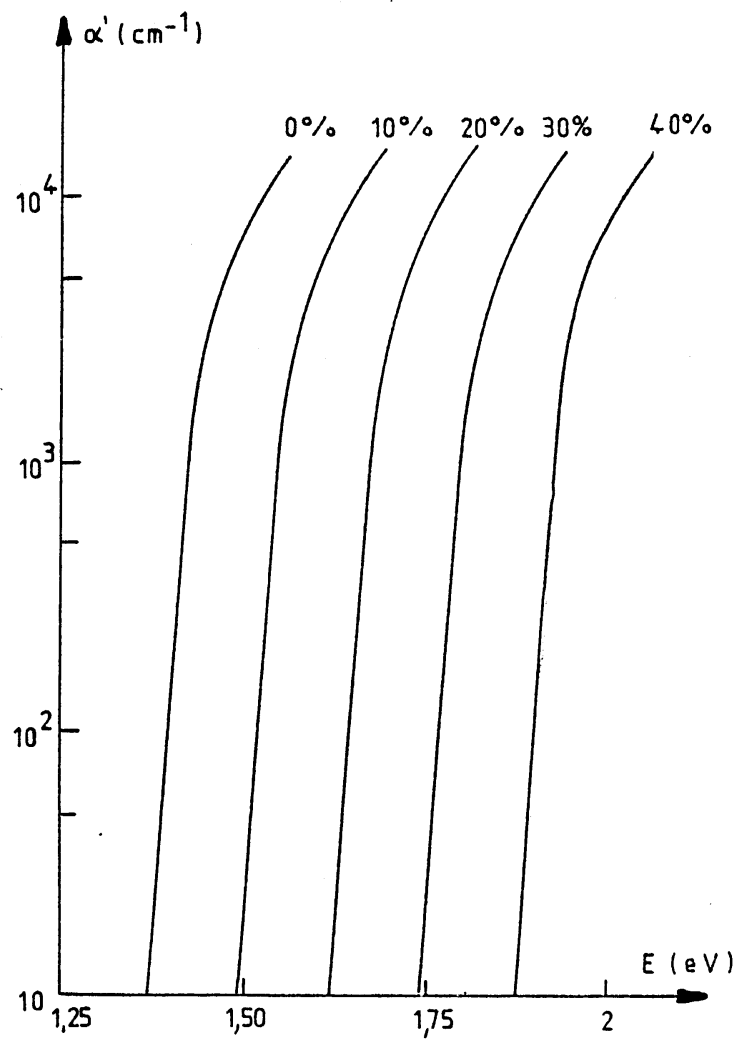

Fig. 4. - Variation du coefficient de création de paires électron-trou avec l'énergie pour les alliages $\mathrm{Ga}_{1-x} \mathrm{Al}_{x} \mathrm{As}$. $x=0$ valeurs expérimentales (Henry et al. [9]), $x \neq 0$ valeurs extrapolées.

[Electron-hole generation coefficient vs. photon energy in $\mathrm{Ga}_{1-x} \mathrm{Al}_{x} \mathrm{As}$. $x=0$ experimental data (from Henry et al. [9]) $x \neq 0$ extrapolated value.]

La partie utile des variations de $\alpha^{\prime}(E)$ concerne la gamme d'énergie $E$ inférieure à $E_{\mathrm{g} \Gamma}$ et peut être décrite par l'équation

$$
\alpha^{\prime}(E)=\alpha_{0}^{\prime} \exp \frac{E-E_{\mathrm{g} \Gamma}}{c k T}
$$

avec $\alpha_{0}^{\prime}=850 \mathrm{~cm}^{-1}$ et $c \# 0,5$.
Le maximum du gain $g_{\max }$ correspond à une énergie $E_{\text {max }}$ telle que

$$
E_{\max }=E_{\mathrm{g} \Gamma}+c k T \ln \left(\frac{1-c}{c} \frac{g_{\max }}{\alpha_{0}^{\prime}}\right) .
$$

Le seuil laser se produit lorsque le maximum du spectre de gain de la couche active permet d'obtenir un gain modal du guide satisfaisant à la condition d'oscillation. Compte-tenu des relations (3) et (7), l'écart des pseudo-niveaux de Fermi correspondant à ce gain est donné par:

$$
\begin{aligned}
& E_{\mathrm{FN}}-E_{\mathrm{FP}}=E_{\mathrm{g} \Gamma}+c k T \ln \left(\frac{1-c}{c} \frac{g_{\max }}{\alpha_{0}^{\prime}}\right)- \\
& -k T \ln (1-c) \text {. }
\end{aligned}
$$

\section{Comportement électrique.}

De façon générale, quelle que soit la structure considérée, le courant qui traverse la structure se décompose en un courant de ruban et un courant dans les zones latérales. Les modèles exposés cidessous concernent la diode VSIS mais peuvent être repris pour la plupart des rubans, notamment en ce qui concerne le calcul du courant de ruban où un modèle analytique général de la double hétérojonction est présenté.

4.1 COURANT DE RUBAN. - Le courant qui traverse la double hétérojonction du ruban se décompose en un courant de recombinaisons dans la couche active et un courant de déconfinement de porteurs aux hétérojonctions.

4.1.1 Courant de recombinaisons dans la couche active. - Le courant de couche active au voisinage du seuil comprend une composante radiative associée à l'émission spontanée et une composante non radiative associée aux niveaux de pièges dans la bande interdite. Compte-tenu de la relation 5, le courant de recombinaisons dans une couche active d'épaisseur $W_{\mathrm{P}}$ a pour expression :

$$
J_{\mathrm{act}}=q W_{\mathrm{P}}\left(\beta \exp \frac{E_{\mathrm{FN}}-E_{\mathrm{FP}}}{k T}+\frac{\Delta n}{\tau_{\mathrm{NR}}}\right)
$$

où $\Delta n$ est l'excès de porteurs injectés pour la polarisation $\left(E_{\mathrm{FN}}-E_{\mathrm{FP}}\right), \tau_{\mathrm{NR}}$ est une durée de vie effective associée globalement aux transitions non radiatives.

La part des mécanismes non radiatifs est évidemment d'autant plus importante que la composition de la couche active se rapproche de celle du point de croisement des vallées $\Gamma, \mathrm{X}, \mathrm{L}$ de la bande de conduction. Les matériaux dont le taux d'aluminium excède $25 \%$ se caractérisent en effet par un dépeuplement de la vallée directe $\Gamma$ au profit des vallées indirectes $\mathrm{X}$ et $\mathrm{L}$ à grandes densités d'états.

Compte-tenu des niveaux d'injection qui caractéri- 


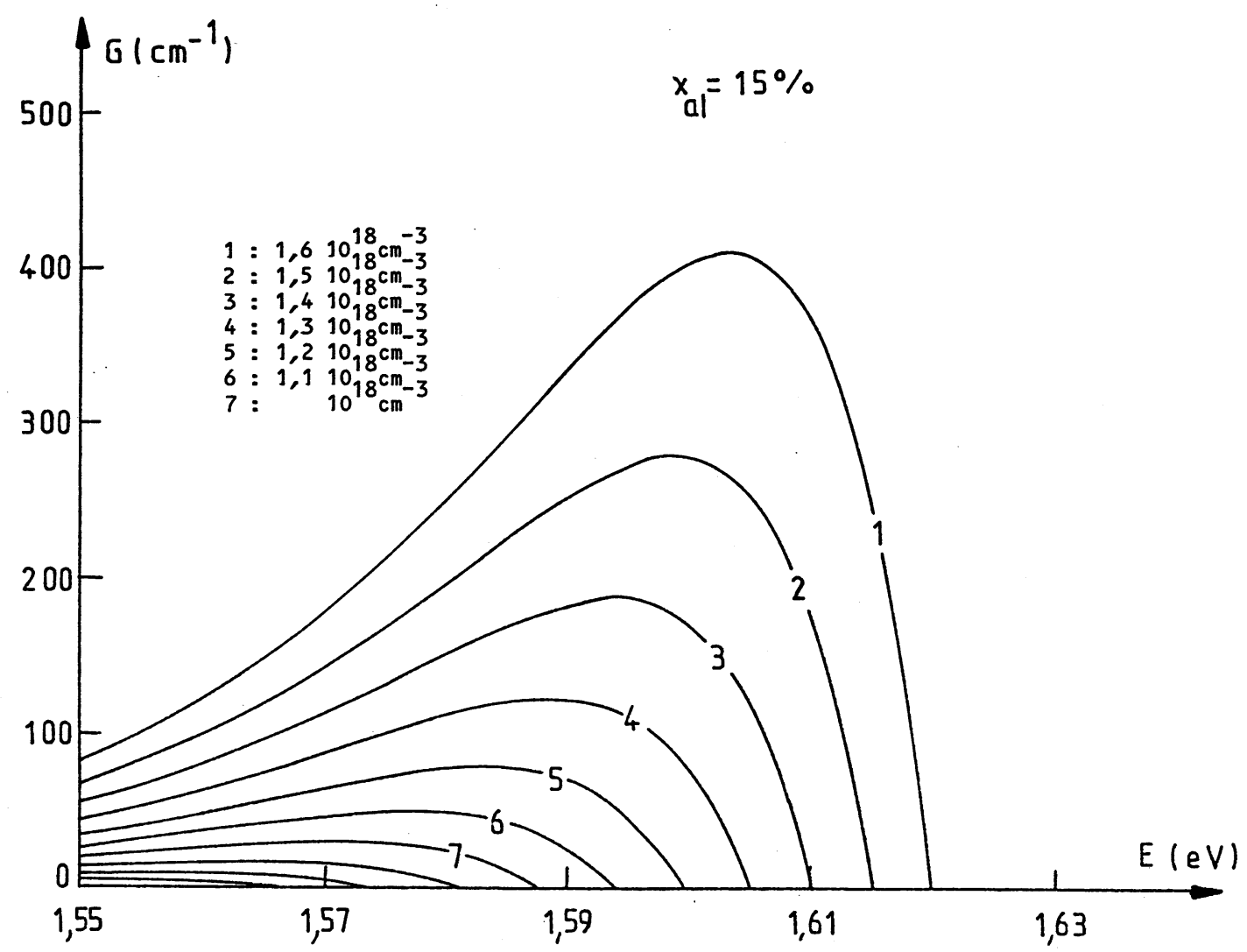

Fig. 5. - Spectres de gain calculés de l'alliage $\mathrm{Ga}_{0,85} \mathrm{Al}_{0,15}$ As à différents niveaux d'injection.

[Calculated gain spectra for $\mathrm{Ga}_{0.85} \mathrm{Al}_{0.15} \mathrm{As}$ at different injection levels.]

sent le régime de fonctionnement au voisinage du seuil, les densités de porteurs sont régies par la statistique de Fermi et satisfont au système d'équations :

$$
\begin{aligned}
n & =p-N_{\mathrm{A}_{\mathrm{P}}} \\
n & =\sum_{j} n_{j} \quad j=\Gamma, \mathrm{X}, \mathrm{L} \\
n_{j} & =\frac{2}{\sqrt{\pi}} N_{\mathrm{C} j} F_{1 / 2}\left(\frac{E_{\mathrm{FN}}-E_{\mathrm{C} j}}{k T}\right) j=\Gamma, \mathrm{X}, \mathrm{L} \\
p & =\frac{2}{\sqrt{\pi}} N_{\mathrm{V}} F_{1 / 2}\left(\frac{E_{\mathrm{V}}-E_{\mathrm{FP}}}{k T}\right)
\end{aligned}
$$

où $F_{1 / 2}$ est l'intégrale de Fermi, $N_{\mathrm{C} j}$ et $N_{\mathrm{V}}$ sont les densités d'états dans les différentes bandes, $N_{\mathrm{AP}_{\mathrm{P}}}$ le dopage de la couche active.

L'écart des pseudo-niveaux de Fermi $\left(E_{\mathrm{FN}}-E_{\mathrm{FP}}\right)$ se déduit des relations (11-14) par le calcul de la fonction inverse de Fermi que l'on approxime généralement par le développement en série de Joyce et Dixon [11].

4.1.2 Courant de déconfinement de porteurs aux hétérojonctions. - Une modélisation complète du courant de ruban doit tenir compte du courant de fuite aux hétérojonctions dû aux porteurs non confinés dans la couche active. Cette composante de courant, généralement négligeable pour les diodes GaAlAs/GaAs émettant dans l'infrarouge, peut devenir prépondérante pour les diodes émettant dans le spectre visible : en effet, dans ce cas, des barrières «idéales" sont d'autant plus difficiles à obtenir que l'efficacité d'injection est moindre et le niveau de polarisation plus élevé. Il peut en résulter une augmentation du courant de seuil rédhibitoire et en tout état de cause une grande sensibilité de celuici à la température, se manifestant par une faible valeur du paramètre caractéristique $T_{0}$.

La figure 6 représente les différentes composantes de courant de la double hétérojonction: $J_{p_{\mathrm{NP}}}$ et

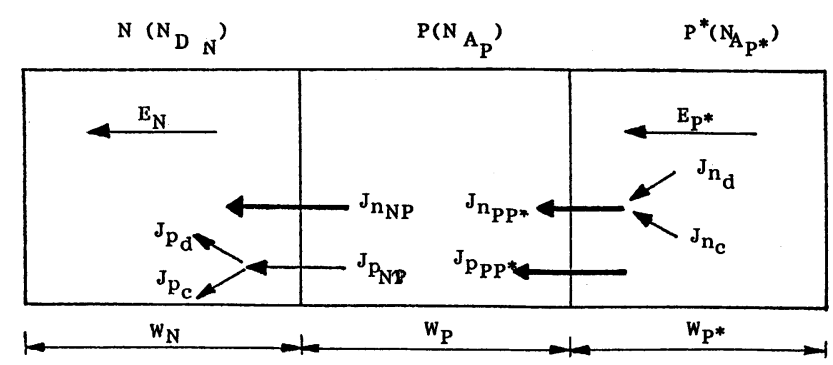

Fig. 6. - Courants d'injection et de fuite dans la double hétérostructure.

[Injection currents and leakage currents in double heterostructure.] 
$J_{n_{\mathrm{PP}}}$ sont respectivement les densités de courant de déconfinement des trous et des électrons aux hétérojonctions NP et PP*. Bien que ces courants aient chacun une composante de diffusion et une composante de conduction, les modèles publiés dans la littérature [12-14] négligent généralement la présence du champ électrique dans les couches de confinement et ne considèrent que les mécanismes de diffusion. Cependant la seule composante de diffusion ne permet pas d'interpréter l'augmentation expérimentale continue des courants de fuite avec le courant total au-delà du seuil laser. Ce phénomène s'explique [15-17] par la présence d'un champ électrique, la prépondérance de la composante de conduction dans le courant de fuite pouvant être mise en évidence par des simulations numériques du comportement électrique de la double hétérojonction, basées sur la résolution du système général des équations des semiconducteurs [8].

Les simulations mentionnées ci-dessus [8] montrent que les pseudo-niveaux de Fermi restent continus aux hétérojonctions et que la statistique de Boltzmann s'applique dans les couches de confinement en raison des niveaux d'injection dans ces régions.

Par suite, connaissant l'écart des pseudo-niveaux de Fermi dans la couche active, la densité de trous $p_{\mathrm{N}}$ injectés à l'hétérojonction NP ou celle des électrons $n_{\mathrm{P}^{*}}$ à l'hétérojonction $\mathrm{PP}^{*}$ se calculent aisément, la position du pseudo-niveau de Fermi des électrons à la limite de la zone de charge d'espace étant donnée pour chaque couche de confinement par la résolution de :

$$
\begin{aligned}
N_{\mathrm{C}} X^{2}+N X^{2}-N_{\mathrm{V}} \exp \frac{E_{\mathrm{FN}}-E_{\mathrm{FP}}}{k T} \times & \\
& \times \exp \frac{-E_{\mathrm{g} \Gamma}}{k T}=0
\end{aligned}
$$

où

$$
X=\exp \frac{E_{\mathrm{FN}}-E_{\mathrm{C} \Gamma}}{k T}
$$

$N_{\mathrm{C}}$ est la densité effective d'états définie par:

$$
\begin{aligned}
N_{\mathrm{C}}=N_{\mathrm{C} \Gamma}+N_{\mathrm{CX}} \exp \frac{E_{\mathrm{g} \Gamma}-E_{\mathrm{gX}}}{k T}+N_{\mathrm{CL}} \times \\
\times \exp \frac{E_{\mathrm{g} \Gamma}-E_{\mathrm{gL}}}{k T}
\end{aligned}
$$

$E_{\mathrm{gi}}(i=\Gamma, \mathrm{X}, \mathrm{L})$ sont les énergies de bande interdite associées à chaque vallée $\Gamma, \mathrm{X}, \mathrm{L}$.

$N$ représente le dopage et est égal suivant la couche à $N_{A_{\mathrm{P}^{*}}}\left(\right.$ couche $\left.\mathrm{P}^{*}\right)$ ou $-N_{\mathrm{D}_{\mathrm{N}}}($ couche $\mathrm{N})$.

Les composantes de diffusion aux hétérojonctions $\mathrm{NP}$ et $\mathrm{PP}^{*}$ s'écrivent respectivement

$$
J_{p_{\mathrm{d}}}=q \frac{D_{p}}{L_{p}} \frac{n_{i \mathrm{~N}}^{2}}{N_{\mathrm{D}_{\mathrm{N}}}} \frac{\exp \left(E_{\mathrm{FN}}-E_{\mathrm{FP}}\right) / k T-1}{\tanh W_{\mathrm{N} / L_{p}}}
$$

$$
J_{n_{\mathrm{d}}}=q \frac{D_{n}^{*}}{L_{n}^{*}} \frac{n_{i \mathrm{P}^{*}}^{2}}{N_{A_{\mathrm{P}}}} \frac{\exp \left(E_{\mathrm{FN}}-E_{\mathrm{FP}}\right) / k T-1}{\tanh W_{\mathrm{P}^{*} / L_{n}^{*}}^{*}}
$$

à partir des densités intrinsèques $n_{i \mathrm{~N}}$ et $n_{i \mathrm{P}}$, des longueurs de diffusion $L_{n}^{*}$ et $L_{p}$, des constantes de diffusion $D_{n}^{*}$ et $D_{p} . D_{n}^{*}$ est une constante effective définie par:

$$
\begin{aligned}
D_{n}^{*}=D_{n \Gamma}+D_{n \mathrm{X}} \exp & \frac{E_{\mathrm{g} \Gamma}-E_{\mathrm{gX}}}{k T}+ \\
& +D_{n \mathrm{~L}} \times \exp \frac{E_{\mathrm{g} \Gamma}-E_{\mathrm{gL}}}{k T} .
\end{aligned}
$$

Compte-tenu des composantes de conduction $J_{p_{\mathrm{c}}}$ et $J_{n_{\mathrm{c}}}$, les courants de déconfinement ont pour expression :

$$
\begin{aligned}
J_{p_{\mathrm{NP}}} & =J_{p_{\mathrm{d}}}+q p_{\mathrm{N}} \mu_{p} E_{\mathrm{N}} \\
J_{n_{\mathrm{PP}}} & =J_{n_{\mathrm{d}}}+q n_{\mathrm{P}^{*}} \mu_{n}^{*} E_{\mathrm{P}^{*}}
\end{aligned}
$$

les champs $E_{\mathrm{N}}$ et $E_{\mathrm{P}^{*}}$ dans les régions $\mathrm{N}$ et $\mathrm{P}^{*}$ se déduisant du courant de porteurs majoritaires $J_{n_{\mathrm{NP}}}$ et $J_{p_{\mathrm{PP}}}$ (Fig. 6).

Comme le courant de ruban est donné par:

$$
J_{\text {rub }}=J_{\text {act }}+J_{p_{\mathrm{NP}}}+J_{n_{\mathrm{pP}}}
$$

il peut être écrit sous la forme :

$$
\begin{aligned}
J_{\text {rub }}=\frac{1}{1-A_{\mathrm{N}}-A_{\mathrm{P}^{*}}}\left(J_{\mathrm{act}}+J_{p_{\mathrm{d}}}\left(1-A_{\mathrm{N}}\right)+\right. \\
\left.+J_{n_{\mathrm{d}}}\left(1-A_{\mathrm{P}^{*}}\right)\right)
\end{aligned}
$$

où

$$
\begin{aligned}
A_{\mathrm{N}} & =\frac{p_{\mathrm{N}} \mu_{\mathrm{PN}}}{n_{\mathrm{N}} \mu_{n_{\mathrm{N}}}+p_{\mathrm{N}} \mu_{\mathrm{PN}}} \\
A_{\mathrm{P}^{*}} & =\frac{n_{\mathrm{P}^{*}} \mu_{n \mathrm{P}^{*}}}{n_{\mathrm{P}^{*}} \mu_{n_{\mathrm{P}^{*}}}+p_{\mathrm{P}^{*}} \mu_{\mathrm{P}_{\mathrm{P}^{*}}}} .
\end{aligned}
$$

La relation (24) permet donc, compte-tenu des relations (18) et (19) d'évaluer le courant de ruban connaissant l'écart des pseudo-niveaux de Fermi dans la couche active.

4.2 COURANT DE DÉCONFINEMENT LATÉRAL AU RUBAN. - Une approche théorique simplifiée de la répartition des lignes de courant dans une structure à ruban VSIS peut être effectuée en considérant un modèle distribué dans la région latérale extérieure au ruban [18]. Elle est schématisée par un ensemble de résistances élémentaires correspondant à la couche de confinement et un ensemble de structures à double hétérojonction élémentaires comme indiqué sur la figure 7.

La répartition latérale de courant est obtenue par résolution numérique de l'ensemble des relations :

$$
V(i)-V(i+1)=\frac{h}{q \mu_{\mathrm{P}^{*}} N_{A_{\mathrm{P}}} t L}
$$




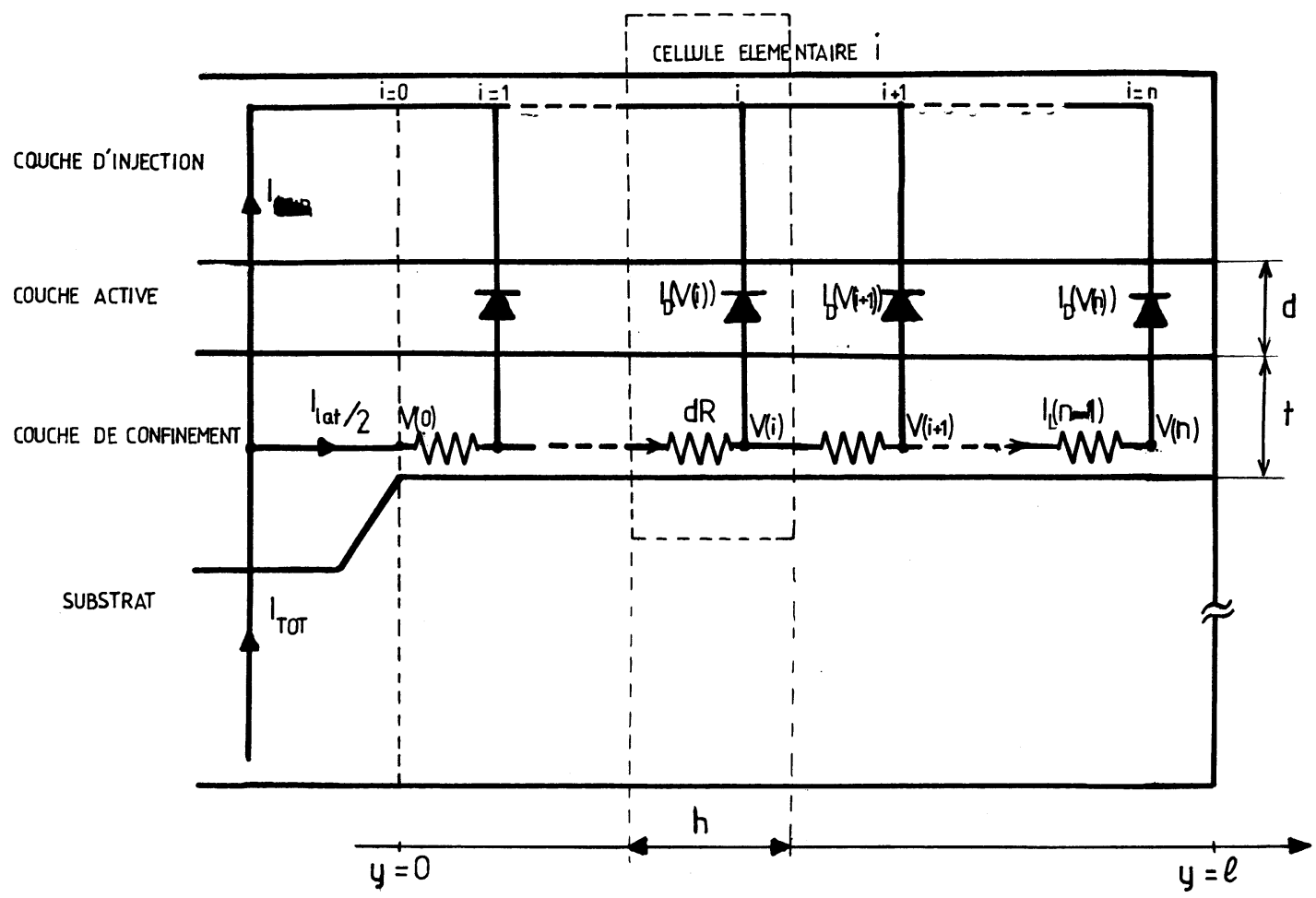

Fig. 7. - Modèle de répartition latérale du courant.

$$
I_{\mathrm{L}}(i)-I_{\mathrm{L}}(i+1)=J(V(i+1)) h L
$$

avec comme conditions aux limites

- en $y=0$

$$
V(0)=V_{\mathrm{a}}
$$

où $V_{\mathrm{a}}$ est la tension appliquée sur le ruban

$$
\text { - en } y=\ell
$$

$$
I_{\mathrm{L}}(n-1)=0
$$

Dans ces expressions $t$ est l'épaisseur de la couche de confinement en dehors du ruban, $\ell$ est la demi largeur de la zone latérale, $h$ est le pas de discrétisation $(h=\ell / n), L$ la longueur de la cavité. La densité de courant $J(V(i+1))$ qui traverse la couche active quand elle est polarisée par $E_{\mathrm{FN}}-E_{\mathrm{FP}}=V(i+1)$ est donnée par les relations (10-14). On néglige dans ce cas les mécanismes de déconfinement transverse aux hétérojonctions, compte-tenu de ce que les diodes latérales sont de moins en moins polarisées au fur et à mesure qu'on s'éloigne du ruban.

5. Méthodologie de détermination de la densité de courant de seuil.

De façon générale, la méthodologie de détermination de la densité de courant de seuil d'une diode laser à ruban peut être décomposée selon les étapes représentées sur la figure 8 .
[Lateral spreading current model.]

i) les pertes et les réflectivités des miroirs déterminent la cavité d'oscillation Perot-Fabry et le gain au seuil dans la cavité $g_{\text {cav }}$,

ii) le guide optique réalisé par la structure confine une fraction de lumière $\Gamma$ dans la couche active : au seuil laser, le gain modal du guide égale $g_{\text {cav }}$ et le gain dans la couche active atteint la valeur $g_{\text {cav }} / \Gamma$,

iii) les propriétés optiques du matériau de la couche active fixent le niveau de polarisation qui permet d'obtenir le gain $g_{\text {cav }} / \Gamma$,

iv) compte-tenu de la polarisation de la couche active, le courant de seuil est la somme de différentes composantes :

- le courant de recombinaisons radiatives et non radiatives dans la couche active,

- le courant de déconfinement transverse aux hétérojonctions,

- le courant de déconfinement latéral de part et d'autre du ruban.

Les modèles qui ont été établis permettent de mettre en œuvre cette méthodologie. Ainsi, pour une diode laser à ruban VSIS, les étapes de calcul du courant de seuil sont les suivantes :

- détermination du gain $g_{\text {cav }}$ d'une cavité de longueur $L$, de réflectivité $R$, de perte $\alpha_{i}$

$$
g_{\mathrm{cav}}=\alpha_{i}+\frac{1}{L} \ln \frac{1}{R}
$$

- détermination du gain $g_{3 \mathrm{~A}}$ selon les étapes décrites par la figure 3 , 
CONDITON D'OSCILLATION

DE LA CAVITE LASER

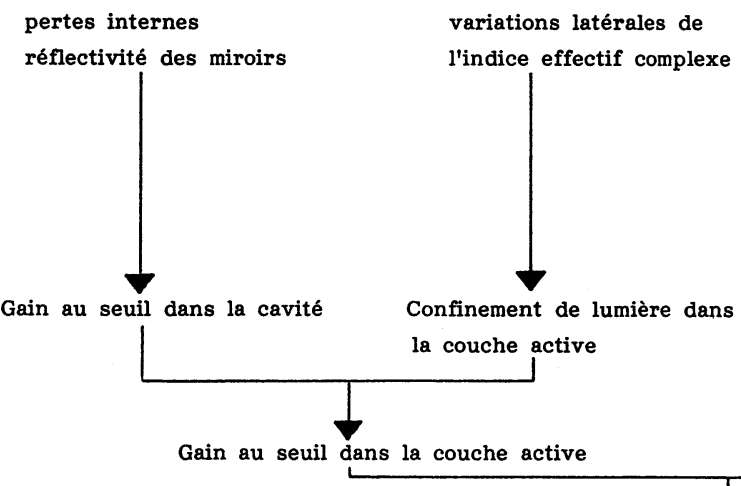

GAIN DU MATERIAU

DE LA COUCHE ACTIVE
COMPORTEMENT ELECTRIQUE DE LA STRUCTURE

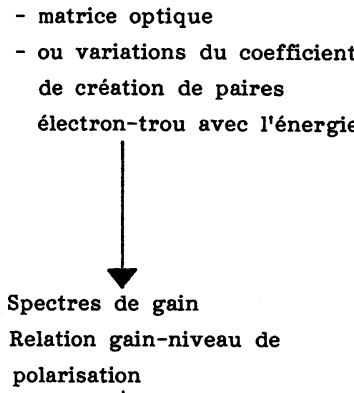

Condition d'injection au seuil

Relation courant total -

niveau de polarisation

Fig. 8. - Méthodologie générale de détermination du courant de seuil.

[General methodology for threshold current determination.]

- calcul de l'écart des pseudo-niveaux de Fermi $E_{\mathrm{FN}}-E_{\mathrm{FP}}$ permettant d'atteindre le gain $g_{3 \mathrm{~A}}$ suivant la relation (9),

- calcul du courant de seuil par la somme :

$$
I_{\mathrm{s}}=J_{\text {rub }} L \cdot W+2 I_{\mathrm{L}}(0) \text {. }
$$

Les logiciels correspondant à chacun des modèles (guide optique - gain du matériau - comportement électrique) ont été développés. Ils peuvent être mis en œuvre individuellement afin d'analyser plus précisément un de ces mécanismes physiques; ils constituent aussi les modules d'un logiciel général de détermination du courant de seuil en s'articulant comme il vient d'être montré autour des paramètres essentiels que sont le gain du matériau et l'écart des pseudo-niveaux de Fermi dans la couche active.

\section{Conclusion.}

Une méthode générale de détermination de la densité de courant de seuil a été présentée. Elle repose sur des modèles analytiques généraux, faciles à mettre en œuvre, qui décrivent :

- le guide optique bidimensionnel, basé sur les variations de l'indice effectif complexe, qui définit le gain que doit atteindre la couche active pour satisfaire à la condition de seuil,

- l'évaluation de la relation gain du matériauniveau de polarisation pour un alliage dont on ne connaît pas théoriquement ou expérimentalement les spectres de gain,

- le comportement électrique complet de la structure dans le cas le plus général où les porteurs se répartissent dans plusieurs vallées de la bande de conduction et où les mécanismes de déconfinement aux hétérojonctions se traduisent par des composantes de diffusion et de conduction dans les couches de confinement.

Cette méthodologie et les modèles qu'elle comporte peuvent être appliqués à différentes diodes à guidage par l'indice présentant par exemple des couches gravées, des rubans enterrés ou nervurés. Par sa généralité, elle peut être également étendue à la modélisation de diodes constituées par des alliages différents de ceux de la famille GaAlAs, que ce soit pour l'étude des diodes GaInAsP/InP ou pour l'évaluation de nouvelles filières telles que les diodes $\mathrm{GaAlInP} / \mathrm{GaAs}$ ou les diodes GaInAsSb/GaSb. Les lois établies pour lier le courant de seuil aux paramètres géométriques et technologiques offrent la possibilité d'analyser le comportement de la structure étudiée, d'optimiser par suite ses performances et d'évaluer ses limites.

\section{Remerciements.}

Les auteurs tiennent à remercier le C.N.E.T. pour le soutien apporté à cette étude. 


\section{Bibliographie}

[1] Yamamoto, S., Hayashi, H., Yano, S., SAKURAI, T., Hijikata, T., Appl. Phys. Lett. 40 (1982) 372.

[2] Streifer, W., Kapon, E., Appl. Opt. 18 (1979) 3724.

[3] Kressel, H., Butler, J. K., Semiconductor Lasers and Heterojunction LEDs (Academic Press) (1977), p. 137-184.

[4] Osinski, M., Polish, M., Adams, M. J., IEEE Proc. Part. I 6 (1982) 229.

[5] Asada, M., Suematsu, Y., IEEE J. Quantum Electron. QE-21 (1985) 434.

[6] Casey, H. C., Stern, F., J. Appl. Phys. 47 (1976) 631.

[7] Stern, F., Phys. Rev. B 3 (1971) 2636.

[8] Lozes-Dupuy, F., Thèse de Doctorat d'Etat Université Paul Sabatier Toulouse (1983).

[9] Henry, C. H., Logan, R. A., Bertness, K. A., J. Appl. Phys. 52 (1981) 4457.
[10] Hutchby, J. A., Fudurich, R. L., J. Appl. Phys. 47 (1976) 3140.

[11] Joyce, W. B., Dixon, R. W., Appl. Phys. Lett. 31 (1977) 354.

[12] Casey, H. C., J. Appl. Phys. 49 (1978) 3684.

[13] Goodwin, A. R., Peters, J. R., Pion, M., Thompson, G. H. B., Whiteaway, J. E. A., J. Appl. Phys. 46 (1975) 3126.

[14] Hayakawa, T., Yamamoto, S., Hayashi, H., SAKURAI, T., HiJIKATA, IEEE J. Quantum Electron. 17 (1981) 2205.

[15] Chen, T. R., Margalit, S., Koren, U., Yu, K. L., Chiu, L. C., Masson, A., Yariv, A., Appl. Phys. Lett. 42 (1983) 1000.

[16] Anthony, P. J., Schumaker, N. E., J. Appl. Phys. 51 (1980) 5038.

[17] Dutta, N. K., J. Appl. Phys. 52 (1981) 70.

[18] NACER, S., Thèse de $3^{\mathrm{e}}$ cycle, Université Paul Sabatier, Toulouse (1985). 